\title{
Monofocal Acute Inflammatory Demyelination Manifesting as Open Ring Sign
}

\author{
-Case Report- \\ Jiro AKImOTO, Nobuyuki NAKAJIMA, Akihiko SAIDA, Jo HARAOKA, \\ and Motoshige KUDO* \\ Departments of Neurosurgery and *Pathology, Tokyo Medical University, Tokyo
}

\begin{abstract}
A 48-year-old woman with a history of viral influenza infection was admitted with rapidly progressive numbness and weakness of the right extremities. On admission, general physical examination revealed no abnormality. Cerebrospinal fluid analysis showed no abnormal findings. Brain computed tomography and magnetic resonance imaging showed an open ring-like enhanced lesion in the white matter of the right parietal lobe with massive perifocal edema. Cerebral angiography showed no tumor staining and thallium-201 single photon emission computed tomography showed no abnormal uptake. The preoperative diagnosis was malignant glioma and partial resection was performed. Histological examination showed perivascular accumulation of small lymphocytes and a large number of macrophages with reactive astrocytes. Phagocytosis of myelin was observed in the macrophages and nuclear fragmentation in the reactive astrocytes. The histological diagnosis was acute inflammatory demyelinating disease. After therapy with methylprednisolone, her neurological symptoms improved gradually and no relapse occurred during 18 months of follow up. Tumor-like masses of demyelination may occupy an intermediate position between acute multiple sclerosis and postinfectious encephalitis. Open ring sign may be a pathognomonic feature of these lesions.
\end{abstract}

Key words: demyelinating disease, magnetic resonance imaging, pathology

\section{Introduction}

Ring-like enhancement on computed tomography (CT) or magnetic resonance (MR) imaging associated with progressive neuronal symptoms is often interpreted as indicating malignant brain tumor or brain abscess. Subsequent differential diagnosis will often be performed exclusively based on such an assumption. ${ }^{5,6,8)}$ However, many other pathological conditions can appear as ring-like enhancement, e.g. tumor-related pathologies such as malignant lymphoma, radiation necrosis, inflammatory diseases such as mycosis, parasitic infection, or tuberculoma, demyelinating diseases such as multiple sclerosis, and cerebrovascular diseases such as old cerebral infarction or cerebral hemorrhage, etc. ${ }^{1,5,10)}$ The first step to adequate treatment is a differential diagnosis considering as many diseases as possible.

We recently treated a patient with acute demye-

Received October 25, 2005; Accepted March 27, 2006 linating disease in whom surgery was performed based on a preoperative diagnosis of brain tumor. Review of the preoperative images after the histological diagnosis was established identified the characteristic open ring sign. ${ }^{10)}$

\section{Case Presentation}

A 48-year-old woman had no significant past history or family history, but developed cold-like symptoms in late January 2004. She visited a local hospital, and was given oral medications under a diagnosis of influenza. She suddenly developed numbness in her right leg on March 8, 2004. She was kept under observation, and the numbness extended to the right arm on March 15, and she also manifested dysgraphia. She developed muscle weakness in the right arm and leg on March 26. She finally presented to our hospital with difficulty in walking on April 3.

She was hospitalized with suspected brain tumor on the basis of CT findings. Her consciousness was clear. She had right flaccid hemiparesis of the arm 

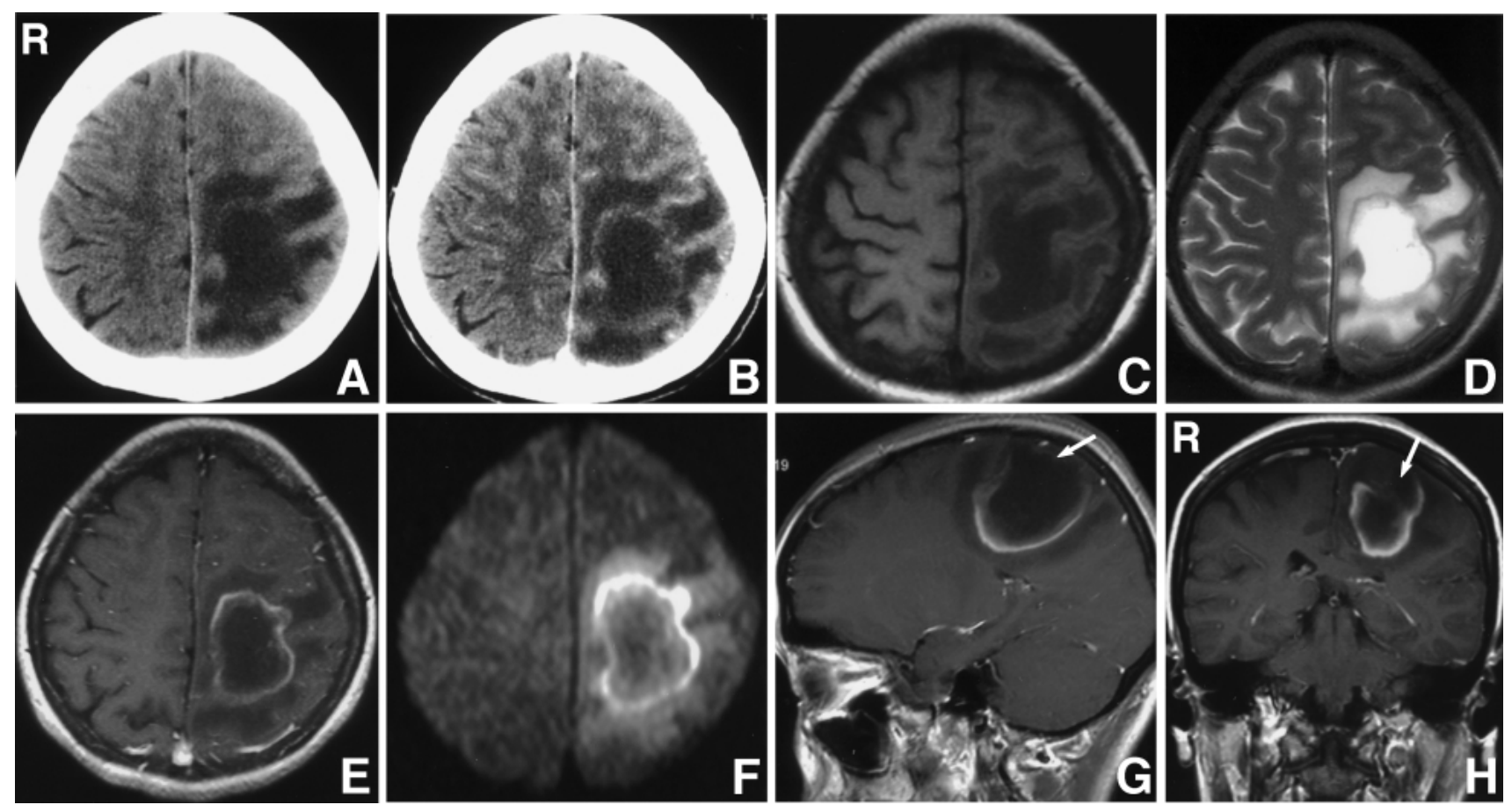

Fig. 1 Preoperative computed tomography scans (A) and with contrast enhancement (B) revealing a hypodense lesion in the left parietal subcortical white matter, with perifocal edema and faint oval-shaped enhancement. $T_{1}$-weighted magnetic resonance (MR) image (C) showing a low intensity and $\mathrm{T}_{2}$-weighted $M R$ image (D) showing a high intensity white matter lesion with wide perifocal edema. Axial (E), sagittal (G), and coronal (H) $\mathrm{T}_{1}$-weighted MR images with contrast medium showing open ring (arrow) perimeter enhancement. Diffusion-weighted MR image (F) showing a hypointensity lesion with a high intensity perimeter ring.

and leg with manual muscle test $4 / 5$ and reduced superficial perception. Blood biochemical examinations found no evident abnormalities including inflammatory findings.

CT revealed a broad low density area in the center of the white matter of the left parietal lobe with blurred ring-like enhancement in the center of the same area with contrast medium. A slight mass effect was found in the posterior horn of the lateral ventricle (Fig. 1A, B). $\mathrm{T}_{1}$-weighted $\mathrm{MR}$ imaging showed a low intensity area in the same region, and $\mathrm{T}_{2}$-weighted MR imaging showed the central part of the lesion as high intensity, with a slightly different signal intensity from the high intensity extending throughout the surrounding white matter (Fig. 1C, D).

MR imaging with contrast medium revealed a ring-like enhancement which seemed to surround the relatively high intensity area on $\mathrm{T}_{2}$-weighted $\mathrm{MR}$ imaging. This area showed strong enhancement in the area bordering the deep white matter, with little enhancement on the cortex of the brain surface on the sagittal image. This feature was also evident on the coronal image. Although the enhancement effect showed a ring-like shape, the ring seemed to open onto the cortex. The peripheral edema was intense, but the mass effect seen in the posterior horn of the lateral ventricle was mild (Fig. 1E-G). Diffusionweighted MR imaging showed the area as high intensity similar to the enhanced area. Furthermore, the surrounding edema-like area also appeared as high intensity (Fig. 1H).

Cerebral angiography was performed because of the possibility of brain tumor. No evident tumor stain was seen but a mass effect was found. Thallium-201 single photon emission computed tomography failed to show any obvious uptake in either early or delayed images. Although an operation was planned because of the possibility of high-grade glioma in the right parietal lobe, emergency surgery was performed on the 3rd day after admission because the patient developed rapidly deteriorating hemiparesis, headache, nausea, and vomiting.

A parietal craniotomy was performed in a prone position. The brain surface showed a flat gyrus. Ultrasonography showed the lesion was hyperechoic. After needle puncture of the focus, $8 \mathrm{ml}$ of yellowish fluid positive for Froin was aspirated. The 

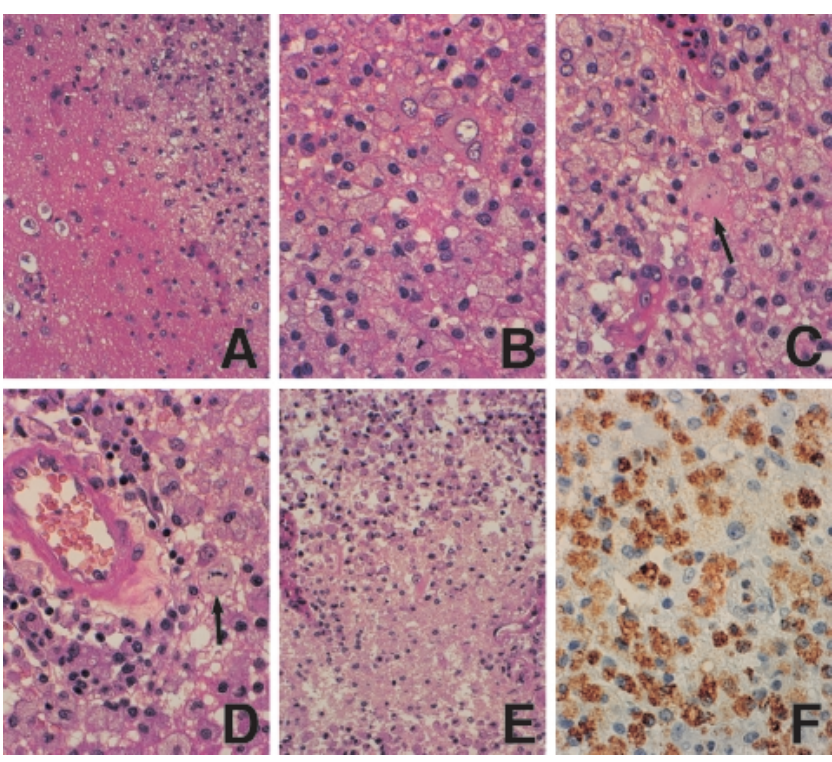

Fig. 2 A-E: Photomicrographs showing the lesion consisted of dense accumulation of foamy macrophages, lymphocytes, and reactive astrocytes with prominent perivascular distribution (A-D), and focal necrosis with reactive astrocytosis (E). Swollen reactive astrocytes, with granular nucleus disruption (Creutzfeldt cell), were seen occasionally (C, D; arrow). Hematoxylin and eosin stain, original magnification A: $\times 100$, BE: $\times 200$. F: Photomicrograph with CD68 immunostaining demonstrating numerous macrophages. Original magnification $\times 200$.

central sulcus was identified with somatosensory evoked potential monitoring, and a corticotomy was performed to remove approximately $2 \mathrm{~cm}$ of the superior parietal lobule. Rapid examination of the yellowish-brown, soft, non-hemorrhagic tissue from the subcortex indicated astrocytoma grade II. Tissue for permanent specimens was extracted piece by piece because the lesion was adjacent to the motor cortex.

Histological examination showed the primary site of the lesion was the subcortical white matter. The cortical structure was well maintained without inflammatory cell infiltration. The chromatin-rich nuclei were unevenly distributed in the white matter, and foamy macrophages showed solid growth with reactive astrocytes containing abundant eosinophilic cytoplasm with thick cell processes. The macrophages were almost uniform in shape, although cells with evident nucleoli as well as binuclear cells and cells with diffuse granular nuclei (Creutzfeldt cells) were found among the astrocytes.
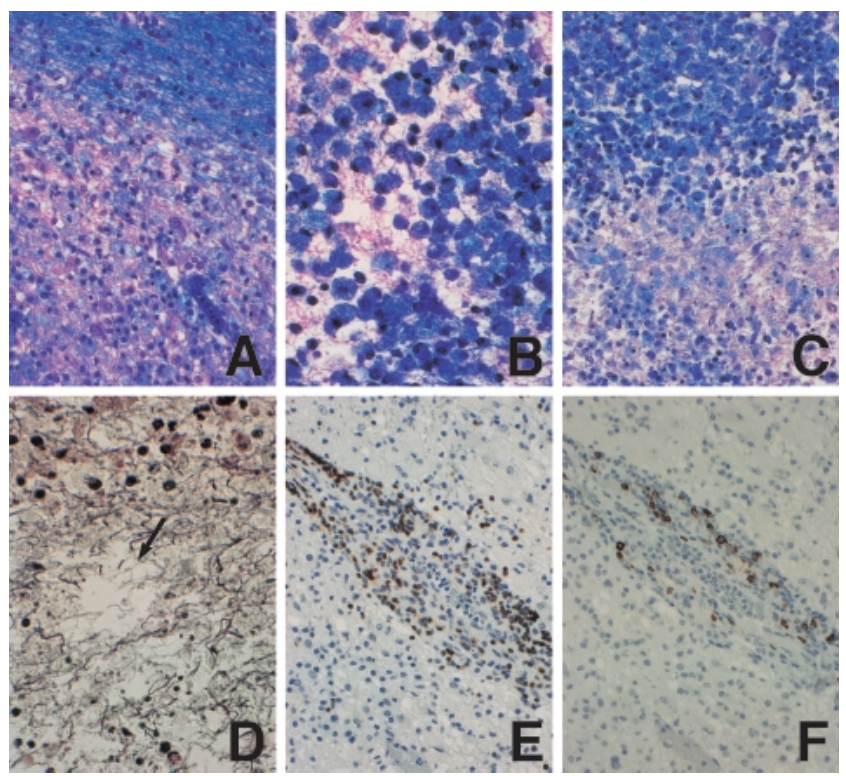

Fig. 3 A-C: Photomicrographs with Luxol fast blue combined with hematoxylin and eosin staining demonstrating a clear boundary between the normal white matter and the demyelinated zone. Numerous macrophages, containing myelin fragments, and focal necrotic changes are also demonstrated. Original magnification $A, C: \times 100$; $B$ : $\times 200$. D: Photomicrograph with Bodian staining demonstrating preservation of axons, but damaged axons in the necrotic area (arrow). Original magnification $\times$ 100. E, F: Photomicrographs with CD3 (E) and CD20 (F) immunostaining demonstrating that most lymphocytes in the perivascular spaces were $T$ cells. Original magnification $\times \mathbf{1 0 0}$.

Regions with extremely low cell density seemed to be microscopic necrotic lesions. No evident change was found in the blood vessel walls, but infiltration by lymphocytes, plasmacytes, etc., was found in surrounding areas (Fig. 2). Luxol fast blue-hematoxylin and eosin staining revealed diffuse demyelinating foci with a clear boundary with the normal white matter preserving the myelin sheath. Phagocytosis of the destroyed myelin by accumulating macrophages was seen in this area (Fig. 3A-C). Bodian staining showed relatively well-preserved axonal structures even in the demyelinated foci, but destruction of axons was also found in the microscopic necrotic areas (Fig. 3D). Lymphocyte infiltration was found for both CD3 and CD20 around the vessels, but CD3 infiltration was dominant (Fig. 3E, F). The histological diagnosis was acute demyelinating lesion. 
Two cycles of steroid pulse therapy (methylprednisolone $1 \mathrm{~g}, 3$ days) were performed. The hemiparesis of the right arm and leg then rapidly improved and the patient managed to walk under observation. Lumbar tap was performed 10 days after the operation. The initial pressure was $170 \mathrm{mmH}_{2} \mathrm{O}$, protein level was $56 \mathrm{mg} / \mathrm{dl}$, glucose level was $67 \mathrm{mg} / \mathrm{dl}$, and no oligoclonal immunoglobulin $G$ was found. MR imaging on discharge found no evident enhancement and the convolutional structure had almost totally recovered. No findings of relapse were found in the 18 months since surgery.

\section{Discussion}

Open-ring imaging sign or white matter crescent sign was first used to describe atypical tumor-like demyelination as a disease characterized by a large enhancement effect localized in the white matter, with the ring-enhancement opening on the side adjacent to the gray matter or the basal ganglia. ${ }^{10)}$ Demyelinating lesions, usually multiple sclerosis, are characterized by small homogeneous ovoid areas, so enhancement often mimics a tumor and is definitely different from conventional demyelinating lesions. ${ }^{1,3,12)}$ The number of cases of demyelinating lesions suspected to be a tumor, and which were treated by surgery including biopsy, has recently increased.1,3,6) The largest series consists of 32 cases,$^{8)}$ and most of the cases included images showing open ring sign..$^{1,6,8,12)}$

Representative terms for this pathology include multiple sclerosis mimicking primary tumor, ${ }^{7)}$ large focal tumor-like demyelinating disease, ${ }^{8)}$ tumefactive demyelinating lesion, ${ }^{1,3,12)}$ and monofocal acute inflammatory demyelination (MAID) ${ }^{6)}$ A disease name should clearly express the pathology, so we believe that MAID is the most suitable name based on the images and clinical findings of the previous cases. The disease is characterized by clinically acute onset and rapid progression..$^{6)}$ Most cases are single onset and show a large open ring sign $4 \mathrm{~cm}$ or more in diameter on imaging, ${ }^{6)}$ usually located in the brain surface area of the frontal lobe or parietal lobe, unlike multiple sclerosis which often occurs in areas such as the deep white matter or the paraventricle. In addition, peripheral edema and relatively mild mass effect for the size of the lesion are common. ${ }^{1,3,12)}$

Many reports have used the term tumefaction and emphasized the importance of differentiation from tumor. Increased diffusion on diffusion-weighted MR imaging, ${ }^{9)}$ reduced blood flow on perfusion MR imaging, ${ }^{5,6)}$ and decreased $\mathrm{N}$-acetyl aspartate and elevated lipid levels on MR spectroscopy are con- sidered to be useful for differential diagnosis. ${ }^{5,6,9,12)}$ Histological features include conventional demyelination findings characterized by macrophage colonies phagocytizing myelin, suggestive of acute onset, perivascular lymphocyte cuffing, or active gliotic change, as well as the presence of necrosis, even accompanied by partial axonal destruction. ${ }^{1,3,11,12)}$ Clinicopathological features require differentiation from the so-called acutely progressive monophasic malignant form of multiple sclerosis such as Marburg disease. ${ }^{8,11)}$ However, the clinical features are different from those of conventional demyelinating diseases because of the good reaction to steroid treatment and good prognosis virtually without relapse. ${ }^{1,6,8,11)}$

The present patient had had influenza 2 months before presentation. Acute demyelinating lesions following infection include the entity acute disseminated encephalomyelitis. However, most reported cases occurred in pediatric patients and showed many differences from typical MAID pathology such as prolongation of severe infectious signs including cerebrospinal fluid or uniform enhancement effects. ${ }^{1-4,6,12)}$ Influenza vaccination performed 10 days before onset is extremely rare in MAID case reports. There may be an allergic phenomenon underlying the disease. ${ }^{8)}$

If the open ring sign was well understood, a high risk operation with craniotomy would probably not be chosen. ${ }^{3,5,6,12)}$ Evaluation of possible demyelinating pathology using MR spectroscopy ${ }^{5,12)}$ or cerebrospinal fluid findings, and discussion with the pathologist will increase the possibility of distinguishing this entity from brain tumor by intraoperative diagnosis. ${ }^{11,12)}$ However, adequate exploration of the pathology of the myelin sheath and the axons is essential for reliable histological diagnosis, and confirmation of the boundary between the normal tissue and the demyelinating foci is particularly useful. ${ }^{11,12)}$ We believe that if confirmation of demyelinating foci is obtained intraoperatively, boundary tissue dissection with normal brain should be performed at safe sites utilizing various functional monitoring. ${ }^{6,8)}$ After the definitive diagnosis is established, the reactivity to steroids is good and the patient can be informed that there is almost no possibility of relapse. ${ }^{6,8)}$

\section{References}

1) Anderson RCE, Connolly ES, Komotar RJ, Mack WJ, McKhann GM, Van Orman CB, Hedlund G, Proctor KAS, Townsend JJ, Walker ML: Clinicopathological review: tumefactive demyelination in a 12-year-old girl. Neurosurgery 56: 1051-1057, 2005 
2) Bennetto L, Scolding N: Inflammatory/post-infectious encephalomyelitis. J Neurol Neurosurg Psychiatry 75 Suppl 1: i22-i28, 2004

3) Dagher AP, Smirniotopoulos J: Tumefactive demyelinating lesions. Neuroradiology 38: 560-565, 1996

4) Dale RC, Sousa C, Chong WK, Cox TCS, Harding B, Neville BGR: Acute disseminated encephalomyelitis, multiphasic disseminated encephalomyelitis and multiple sclerosis in children. Brain 123: 2407-2422, 2000

5) Ernst T, Chang L, Walot I, Huff K: Physiologic MRI of a tumefactive multiple sclerosis lesion. Neurology 51: 1486-1488, 1998

6) Gutrecht JA, Berger JR, Jones HR, Mancall C: Monofocal acute inflammatory demyelination (MAID): A unique disorder simulating brain neoplasm. South Med J 95: 1180-1186, 2002

7) Hunter SB, Bellinger WE, Rubin JJ: Multiple sclerosis mimicking primary brain tumor. Arch Pathol Lab Med 111: 464-468, 1987

8) Kepes JJ: Large focal tumor-like demyelinating lesions of the brain: intermediate entity between multiple sclerosis and acute disseminated encephalomyelitis? A study of 31 patients. Ann Neurol 33: 18-27, 1993
9) Kuker W, Ruff J, Gaertner S, Mehnert F, Mader I, Nagele T: Modern MRI tools for the characterization of acute demyelinating lesions: value of chemical shift and diffusion-weighted imaging. Neuroradiology 46: 421-426, 2004

10) Masdeu JC, Quinto C, Olivera C, Tenner M, Leslie D, Visintainer P: Open-ring imaging sign: highly specific for atypical brain demyelination. Neurology 54: 1427-1433, 2000

11) Sugita Y, Terasaki $M$, Shigemori $M$, Sakata K, Morimatsu M: Acute focal demyelinating disease simulating brain tumors: histopathologic guidelines for an accurate diagnosis. Neuropathology 21: 25-31, 2001

12) Tan HM, Chan LL, Chuah KL, Goh NSS, Tang KK: Monophasic, solitary tumefactive demyelinating lesion: neuroimaging features and neuropathological diagnosis. Br J Radiol 77: 153-156, 2004

Address reprint requests to: J. Akimoto, M.D., Department of Neurosurgery, Tokyo Medical University, 6-7-1 Nishishinjuku, Shinjuku-ku, Tokyo 160-0023, Japan. e-mail: akimoto-nsu@umin.ac.jp 